\title{
Scientifically Substantiated Guidelines for Physico-mathematical Modelling of Laser Surface-treatment of Wear-resistant Implants for Human Joint Replacements
}

\author{
Uldis Zaimis ${ }^{1,2, a}$, Sharif E. Guseynov ${ }^{1,2,3, b}$ \\ ${ }^{1}$ Institute of Fundamental Science and Innovative Technologies, Liepaja University, Liepaja LV-3401, Latvia; \\ ${ }^{2}$ Faculty of Science and Engineering, Liepaja University, Kr. Valdemar Street 4, Liepaja LV-3401, Latvia; \\ 3"Entelgine" Research \& Advisory Co., Ltd., Kleistu Street 2-53, Riga LV-1067, Latvia; ${ }^{a}$ uldiss.z@inbox.lv, \\ sh.e.guseinov@inbox.lv
}

\begin{abstract}
This paper presents the main results from a set of guidelines on the physico-mathematical modelling of laser surface-treatment of wear-resistant implants for human joint replacements. These guidelines contain synthesized and documented procedure, theoretical and practical recommendations, which are provided by researchers in the Nanomaterials Laboratory under the Institute of Fundamental Science and Innovative Technologies, Liepaja University. The present paper does not attempt to cover all aspects of physical and mathematical modelling, but draws together many key aspects concerning theoretical and practical difficulties, in the overcoming of what researchers of the Nanomaterials Laboratory, in particular, the authors of the present paper, have sufficient skills.
\end{abstract}

Keywords: wear-resistant implant, human joint replacement, laser surface-treatment, physico-mathematical modelling, scientifically substantiated guidelines.

\section{INTRODUCTION}

Modern medicine widely uses artificial materials to replace damaged tissues, bones and organs. Depending on their purpose, the implants involved into the body should be gradually replaced with a living tissue and / or to function for a long time period.

The tissue composition of the human bone has a complex structure. The mechanical strength of bone tissue is based on spatial arrangement of the structural components of bones - bone plates. The composition of the bone includes an organic component - collagen $(\approx 25 \%)$, inorganic component - calcium phosphates $(\approx 65 \%)$, water $(\approx 10 \%) \quad([1])$. Artificial materials claiming the role of implants must meet the requirements dictated by the composition and properties of human bone tissue. The main requirements for artificial materials for implantation are resistance to corrosive media and biomechanical compatibility. In addition, the implant material must also posse some mechanical properties, of which the most prestigious are hardness, tensile strength and modulus of elasticity. Since the response of any material to repeated cyclic loads depends on the fatigue strength of the material, it is this property that determines the continues usage time of the implant ([2]). Because of the fact that the human body is an aggressive environment for implants, especially those made of metals, when developing new implants, it is necessary to take into account not only their functional characteristics, but also their interaction with the biological environment in where they will be used in. Low wear resistance and low corrosion resistance of metal implants in the corrosive liquid medium of the human body promote the release of metal ions into the body. It has been found ([3]) that $\mathrm{Ni}^{2+}, \mathrm{Co}^{3+}, \mathrm{Al}^{3+}, \mathrm{Cr}^{3+}$ ions cause allergic and toxic reactions in the body, and therefore materials used as implants should not have toxicity or should have reliable protective coatings on surfaces that prevent Release of metal ions in the tissues of the human body. For small-size implants, pitting corrosion also poses a risk: for example, the functional integrity of the vascular walls can be destroyed by the presence of only one minor deepen.

On the surface and boundaries of the implant and soft tissues of the body undergo chemical and biological processes, carried out, in particular, on the cell-matrix and nanoscale level. The search for new biocompatible materials with nanostructured characteristics has been implementing according to the biomimetic approach, according to which artificial nanomaterials mimic the properties of biomaterials from living nature. Qualitative progress 
in this area is possible only based on interdisciplinary research in medicine, chemistry, biology, materials science, physics and mathematics. Since the 60s of the XX century, specialists in the areas listed above work closely together to understand the complex processes of interaction of body cells with the foreign surface of the implanted material. Biologically active properties of the interface between the tissues of the body and the implanted material reviewed in correlation with its specific properties. The chemical composition of the surface, its physical and chemical characteristics, roughness and morphology, the determination of the activity of various cellular structures acting separately and synergistically. The relationship between the physical and chemical properties of the surface and the cellular response is far from to be clear (for instance, see [1]-[7] and respective references given in these). A firm understanding of the mechanism of this connection will lead to the creation of a new generation of materials that can be more effectively and successfully involved into the human body for therapeutic purposes. The classical concept of bioinert or bioactive materials with the development of materials science and chemical science will shift to the field of creating more advanced biomaterials that could react or facilitate the implementation of various reactions with the biological environment depending on the state of the surface of the implanting material.

Without overlooking of the comparative analysis of the properties, advantages and disadvantages of artificial materials (for instance, see [1], [8], [9]), used in modern implantation surgery, in particular, in endoprosthetics, we list only the types of most frequently used materials, which can be toxic (surrounding tissues die off on contact), bioinert (non-toxic, but biological inactive) and bioactive (non-toxic, fused with bone tissue): composite biomaterials, bioglass and bioceramics; alloys of titanium, zirconium and magnesium. We will investigate only some aspects of laser surface treatment of titanium implants in this paper.

\section{BRIEF OVERVIEW OF THE BASIC PROPERTIES OF TITANIUM IMPLANTS}

For the first time, the concept of the use of industrially pure titanium and its alloys as implants was put forwarded by Swedish therapist P.-I. Branemark and his scientific group (for instance, see [10], [11] and [12]): from the experimental work carried out in 1952-1969 it is showed the phenomenon of ingrowth of a titanium structure into living bone tissue (now this phenomenon is called osseointegration), and between the implant surface. The fibrous tissue does not form a fibrous or cartilaginous tissue. Since 1965, titanium implants have been used as bio- and mechanically compatible material for the manufacture of implants. Nowadays, numerous fundamental and clinical studies in vitro and in vivo have shown that industrially pure titanium, titanium-aluminum alloy, titaniumvanadium alloy, and nickel-titanium alloy having shape memory effect have such exceptional qualities as high mechanical strength, low cycle fatigue, plastic, high biocompatibility and biotolerance, antiallergenicity and lack of toxicity, high corrosion resistance due to the formation on the surface of a passivating oxide layer, low thermal conductivity, small coefficient of linear expansion, relatively low specific gravity, the smallest difference between the moduli of elasticity and bone. All listed properties of titanium and its alloys are based on their microstructures. As shown in [13], in order to reduce the modulus of elasticity of industrially pure titanium, the most effective alloying elements for the additive are niobium $(\mathrm{Nb})$, zirconium $(\mathrm{Zr})$, molybdenum (Mo), tantalum (Ta). Nowadays, in the production of implants, industrially pure titanium grades BT1-0 and BT1-00 are used (for instance, see [8], [14]) from so called "commercially pure" titanium grades Ti-6Al4V, Ti-6Al-7Nb; BT6, Grade-1 $\div$ Grade-4, Grade-2H, Grade-6, Grade-7, Grade-7H, Grade-9, Grade-11 $\div$ Grade-16, Grade-16H, Grade-17 $\div$ Grade-26, Grade$26 \mathrm{H}$, Grade- $27 \div$ Grade- 38 .

It should be noted in conclusion that despite the very high mechanical properties of titanium alloys containing aluminum (Al) and vanadium (V), one should clearly know and distinguish the field of their application, because these elements can accumulate in the human body tissues and, due to the corrosive effect of biological fluids, cause acute allergies and have toxic effects. It is important to note that titanium implants, in spite of the above unique properties and advantages, still: (a) undergo abrasive wear, which often leads to loss of the implant; (B) can cause intolerable pain and pathogenicity due to the fact that wear products are deposited on the human body tissues. Therefore, the unresolved and actual problem is the improvement of existing and development of new methods and technologies for the production of titanium implants and the treatment of their surfaces in order to enhance osseointegration contact, as well as proper biomechanical, chemical-biological (cellmatrix and nanoscale Level) of contacts between the implant and living human bone tissue.

\section{LASER TREATMENT OF THE SURFACE OF TITANIUM IMPLANTS \\ A. Relevance and status of the issue}

The state of the surface of titanium implants plays a decisive role in cell-cell adhesion. The interaction between the biological environment (hard and soft tissues, blood, body fluids and serum, etc.) and the implant occurs on its surface, and the biological response from the living tissue depends on a variety of surface properties, such as chemical composition, purity, texture and topography, surface energy, corrosion resistance, and the tendency to 
denaturalization of surrounding proteins. Nowadays, developed various methods of surface treatment of titanium implants to improve their biocompatible properties and to accelerate the healing process of implants. These methods are based on morphological or biochemical modification of physical, chemical and mechanical properties, in particular, changes in surface energy and surface charge, chemical composition and surface printing of titanium implants. Approximately 20 years ago a new perspective direction arose related to the use of laser technology for surface treatment of biocompatible metallic materials, in particular titanium implants, in order to improve the tribological, biocompatible and corrosive properties of their surfaces. Theoretically, laser processing technology can possibly solve all the above tasks of modifying the complex of surface properties of titanium implants. Application of laser processing technology provides high spatial resolution and high processing speed, and does not affect the bulk properties of the processed material. Recent works (for instance, see [15]-[17] and appropriate references given in these) show that laser treatment of implant surfaces from titanium leads to the formation of a developed surface morphology characterized by a cellular structure, an increase in roughness and the formation of an inert oxide layer, which is necessary for improving the biocompatibility of titanium implants, in particular, enhancing the adhesion of living cells of different scales. In subsection conclusion, in the context of discussing the laser treatment advantages of implant surfaces, let us dwell only on the results of the work [16]: a group of Italian scientists carried out a rather curious experiment with the aim of: (a) studying histomorphometric and biomechanical characteristics (BIC-bone-implant contact; RTV - reverse torque value) of titanium implants, which underwent laser treatment; (B) comparing the ability of laser irradiation to stimulate osseointegration with a similar ability of titanium implants that have been mechanically processed. The results of the experiment showed that RTV for titanium implants treated with a laser was almost 3 times higher than for those that received mechanical treatment; BIC titanium implants treated with a laser proved to be at least $30 \%$ better than BIC titanium implants, machined.

B. Mathematical model in terms of spherical trigonometry for describing the transfer of laser radiation during surface treatment of the titanium head of the endoprosthesis

Medical practice, especially in countries formerly part of the USSR, shows that repeated prosthetics of the hip joint of a person is associated with big problems (including due to the relatively high cost of surgery, inaccessible to many needy patients). In many cases, the implementation of such surgical operations becomes virtually impossible and, therefore, the life of the hip endoprosthesis is extremely important for the patient, especially at a young age. Modern joint endoprosthesis consists of an acetabular cup, a polyethylene liner; an endoprosthesis stem and a ball-shaped head (see Fig. 1). Titanium heads of the human hip joint endoprosthesis, in terms of the nature and magnitude of their load, are in extreme conditions ([6], [18]), and therefore, the problem of improving the accuracy and quality of processing the most significant part of the endoprosthesis - the spherical surface of the head - is one of the most actual problems in modern endoprosthetics. Completely modern equipment for laser processing of materials, which has a Laboratory of Nanomaterials at the Institute of Basic Sciences and Innovative Technologies of the University of Liepaja (Nanomaterials Laboratory under the Institute of Fundamental Science and Innovative Technologies, Liepaja University), has a fixed headsource of laser radiation.
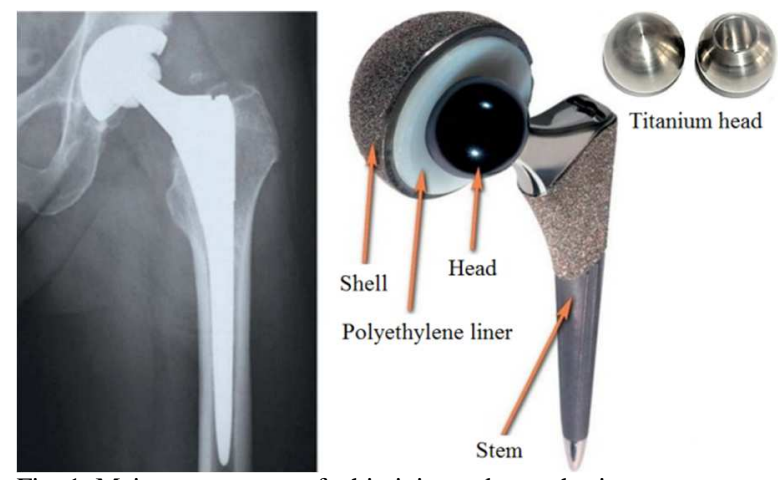

Fig. 1. Main components of a hip joint endoprosthesis.

Therefore, in order to carry out a high-precision uniform laser treatment, the endoprosthesis head, (which has a surface with a $270^{\circ}$ angle of the spherical segment) (see Fig. 2, [6]), it is necessary to rotate the endoprosthesis head properly: the endoprosthesis head is fixed with a special holder in the vacuum chamber laser equipment, and this device can rotate the head of the endoprosthesis along two perpendicular axes with constant cycles per unit time (for each rotation axis its constant cycle).

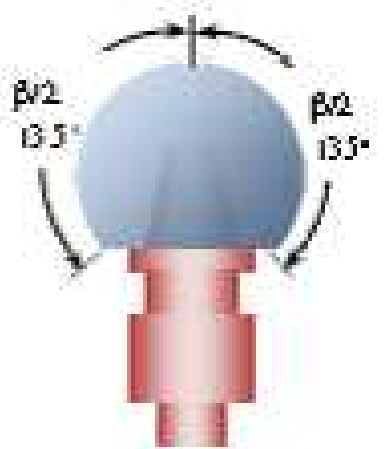

Fig. 2. The head of the endoprosthesis, which has a surface of $270^{\circ}$-angle of the spherical segment. 
If the 3D laser-imaged material were a polyhedron, then the necessary calculation formulas for high precision and high-quality laser processing of this polyhedron can be derived from the corresponding mathematical model constructed in the Cartesian coordinate system. Obviously, because of the fact that: (a) the source of laser radiation on the existing laser equipment is stationary; (B) the endoprosthesis head has a spherical surface with a $270^{\circ}$ angle of the spherical segment being treated; (C) a two-coordinate holder can rotate the endoprosthesis head only in cycles, it is necessary to conduct studies using spherical trigonometry (for instance, see [19], [20]): as the initial construction of the required adequate mathematical model in a Cartesian coordinate system with subsequent derivation from it of the necessary calculation formulas and the "mechanical" translation of the equations and conditions of the already constructed mathematical model, as well as the resulting computational formulas from the Cartesian coordinate system to the spherical coordinate system, will not provide a correct description of the process of precise, uniform and qualitative treatment of the spherical surface of the head of the hip joint endoprosthesis. In such a case, the work of the exposed (ie, emitting laser radiation) is performed using the Euclidean metric as a measure of closeness between two points (defined in the 3D Cartesian coordinate system) and the work of the affected object. Reception of laser radiation on the spherical surface of the rotating head of the endoprosthesis. The affected object is carried out using a spherical metric as a measure of proximity between two points a priori defined in the $3 \mathrm{D}$ spherical coordinate system.

Consider the problem of transferring laser radiation in a parallel vertically inhomogeneous layer of a 3D spherical region (Fig.2), and, for simplicity, assuming the radius equal to 1 . We make the following assumptions:

- on the top border $z=0$ of analyzed layer with width of $H$ and angle $\alpha_{0}=\arccos \left(\mu_{0}\right)$ falls laser power $F$ laser irradiation in azimuthal plane $\beta_{0}, s_{0}=\left\{\alpha_{0}, \beta_{0}\right\}$;

- inside the layer under consideration and at its boundaries there may be diffusion sources of radiation;

- the underlying surface at the lower boundary of the layer under consideration reflects the entire irradiation within a given law

- transfer of irradiation occurs without changing the frequency.

The problem consists in determining the intensity of the multiply incident laser radiation inside the layer $0 \leq z \leq H$, and also in determining the law of the reflected and missed laser radiation.
As was mentioned above, the direction of propagation of multiple laser radiation at an arbitrary point of the layer $0 \leq z \leq H$ It is necessary to describe with spherical coordinates: azimuth $\varphi \stackrel{\text { def }}{\equiv} \frac{2}{5} \cdot(4 \cdot \beta-\pi) \in[0,2 \cdot \pi]$ where $\beta \in\left[\frac{\pi}{4}, \frac{3 \cdot \pi}{2}\right]$ characterizes the corresponding azimuth angle for the spherical segment of Fig. 2, and the zenith angle $\alpha \in[0, \pi]$, measured from the direction of the inner normal to the plane $z=0$ with unit vector $\vec{n}_{0}$, on $O Z$. In this case, as $\varphi=0$ accept the azimuth of the external laser beam $\beta_{0}=0$. Then direction $0^{\circ} \leq \alpha=\alpha^{+}<90^{\circ}$ will be according the descending / missed radiation, and the direction $90^{\circ}<\alpha=\alpha^{-} \leq 180^{\circ}$ will correspond to the ascending / reflected radiation. We denote by: $\mu \stackrel{d e f}{\equiv} \cos (\alpha), \quad \mu^{+} \stackrel{\text { def }}{\equiv} \cos \left(\alpha^{+}\right) \in(0,1]$, $\mu^{-} \stackrel{\text { def }}{\equiv} \cos \left(\alpha^{-}\right) \in[-1,0)$. Then the sets $D^{+}$and $D^{-}$, are defined as

$$
\begin{aligned}
& D^{+} \stackrel{\text { def }}{\equiv}\{(\mu, \varphi): \mu \in[0,1], \varphi \in[0,2 \cdot \pi]\} ; \\
& D^{-} \stackrel{\text { def }}{\equiv}\{(\mu, \varphi): \mu \in[-1,0], \varphi \in[0,2 \cdot \pi]\},
\end{aligned}
$$

will characterize the hemispheres of the directions of the descending / missed and ascending / reflected radiations. In this case, obviously, the set $D \equiv D^{+} \cup D^{-}$is a single sphere of directions for the transfer of laser radiation. If we denote the required intensity of the multiply-incident laser radiation inside the layer $0 \leq z \leq H$ in direction $s=\{\alpha, \varphi\}$ through $I_{R I}(z, \mu, \varphi)$, so it can be written:

$$
\begin{array}{r}
\mu \cdot \frac{\partial I_{R I}(z, \mu, \varphi)}{\partial z}+\left(f_{t}(z)+f_{a}(z)\right) \cdot I_{R I}(z, \mu, \varphi)= \\
f_{r}(z) \cdot \int_{D} I_{R I}(z, \tilde{\mu}, \tilde{\varphi}) \cdot \rho(z, \omega) d \tilde{\mu} d \tilde{\varphi}+F(z, \mu, \varphi),(1) \\
\left.I_{R I}(z, \mu, \varphi)\right|_{\partial D_{0}}=g_{0}(\mu, \varphi)+F(0, \mu, \varphi) \cdot \delta\left(s-s_{0}\right),(2) \\
\left.I_{R I}(z, \mu, \varphi)\right|_{\partial D_{H}}=g_{H}(\mu, \varphi)+ \\
\frac{a \cdot \mu^{2}}{2 \cdot \pi} \cdot \int_{D^{+}} \tilde{\mu} \cdot I_{R I}(H, \tilde{\mu}, \tilde{\varphi}) d \tilde{\mu} d \tilde{\varphi},(3)
\end{array}
$$

where

- function $f_{t}(z)$ and $f_{a}(z)$, through which the transmission and absorption coefficients of the laser radiation are designated, respectively, mean the physical characteristics of the surface of the endoprosthesis head; 
$-\quad \omega \stackrel{\operatorname{def}}{\equiv} \cos (\gamma), \quad \int_{D} \rho(s, \tilde{s}) d \tilde{s}=1, \quad \int_{D} \rho(\omega) d \tilde{s}=1$, $\int_{-1}^{+1} \rho(\omega) d \omega=\frac{1}{2 \cdot \pi}$, where the transfer angle $\gamma$ from direction $\tilde{s}=\{\tilde{\mu}, \tilde{\varphi}\}$ in direction $s=\{\mu, \varphi\}$ is defined by the equation $\gamma=\arccos (\mu \cdot \tilde{\mu}+\sin (\alpha) \cdot \sin (\tilde{\alpha}) \cdot \cos (\varphi-\tilde{\varphi})) ;$

- in geometries $\{z, \alpha, \varphi\}$ and $\{z, \alpha\}$ boundary conditions (2) are determined on the following sets, respectively:

$$
\begin{aligned}
& \partial D_{0}=\left\{(z, \mu, \varphi): z=0, s \in D^{+}\right\}, \\
& \partial D_{0}=\{(z, \mu): z=0, \mu \in(0,1]\} ;
\end{aligned}
$$

- in geometries $\{z, \alpha, \varphi\}$ and $\{z, \alpha\}$ boundary conditions ( 3 ) are determined on the following sets, respectively:

$$
\begin{gathered}
\partial D_{H}=\left\{(z, \mu, \varphi): z=H, s \in D^{-}\right\}, \\
\partial D_{H}=\{(z, \mu): z=H, \mu \in(-1,0]\} .
\end{gathered}
$$

As in the boundary conditions (3) the required function-intensity itself is present, then the proposed mathematical model (1)-(3) is a specific type of inverse boundary value problem for determining the function $I_{R I}(z, \mu, \varphi)$ in spherical geometry field $\{z, \mu, \varphi\}=[0, H] \times[-1,+1] \times[0,2 \cdot \pi]$, in which all parameters defined in (1)-(3), but function $I_{R I}(z, \mu, \varphi)$, are assumed to be given, including the laws of the reflected and missed by the layer of laser radiation. We recall that in the complete formulation of the problem formulated above (see, after the enumerated assumptions in this subsection), it was also required to determine the laws of the reflected and missed laser radiation. Unfortunately, the imposed restriction on the size of this article does not allow us to formulate two more mathematical models for determining, respectively, the law of reflected radiation and the law of laser radiation missed by the layer under consideration: both models have a more complex structure than model (1)-(3). In addition, it should be noted that the authors of this paper developed a numerical algorithm based on a combination of the Tikhonov regularization method ([21]) and the method of characteristics ([22]-[24]), which makes it possible to realize the mathematical model (1)-(3).

\section{IV.BRIEF SUMMARY OF OUTCOMES OF EXPERIMENTS}

The following results of four experiments on laser surface treatment of biocompatible titanium implants are briefly summarized taking into account the model (1)-(3), which has been implemented using the application package MathCAD 14 (version
14.0.0.163) on the basis of a combination of the Tikhonov' regularization method and the method of characteristics. Alongside with a brief results overview for each experiment, there are given the following specifications: the medium type (MEDIUM) with indication of pressure $(p)$, in which the laser treatment was performed; the type of laser used (TYPE); the wavelength of laser emission $(\lambda)$; the frequency of laser emission $(f)$; the duration of laser pulse $(t)$; the energy density of laser beam $(q)$.

Experiment 1. MEDIUM=high vacuum $\left(p=2 \cdot 10^{-4} \mathrm{~Pa}\right) ; \quad$ TYPE $=\mathrm{Nd}: \mathrm{YAG}$ laser $\quad(\mathrm{ns}) ;$ $\lambda=1.064 \mu \mathrm{m} ; \quad f=30 \mathrm{kHz} ; \quad t=300 \mathrm{~ns} ;$ $q=25 \mathrm{~J} / \mathrm{cm}^{2}$ (energy in laser pulse: $E=2 \mathrm{~mJ}$ ). Brief results overview: no melting occurs on the titanium surface (Ti-surface) for the given single laser pulse; melting traces are observed only where the number of laser pulses is equal to or more than 240 , and from this moment the process of capillary waves emergence begins due to variations in surface thermal stresses. As a result of this process, a characteristic surface relief is formed in the form of ledges, hollows, flutes, etc. An increase in the magnitude of the relief leads to a decrease in the reflective ability of the metal surface, which leads to absorbed laser energy increase. At more than 600 pulses, the microstructure forming of the Ti-surface slows down and islands with a smooth surface containing microcracks and pores are formed due to thermal stresses. At more than 1500 pulses, the Ti-surface morphology completely changes: the central zone becomes completely smooth and flat with a polyhedral microstructure sized to several $\mu \mathrm{m}$ and oriented towards the boundary of the laser crater. Any further increase of the laser pulse number leads to a gradual growth of structural non-uniform areas up to several tens of $\mu \mathrm{m}$, and the modified layer depth becomes equal to $1.5 \mu \mathrm{m}$ for the 1500 number of pulses, and $4.5 \mu \mathrm{m}$ for the 21000 number of pulses. The height of ledges depending on the number of pulses increases from $0.5 \mu \mathrm{m}$ (600 pulses) to $3 \mu \mathrm{m}$ (21000 pulses). At absorbed energy depth around $1 \mu \mathrm{m}$ a molten zone is formed and after its crystallization its surface becomes smoother than the original Ti-surface. Deeper melted layers crystallization leads to profiled dendritic relief patterning. This happens due to surface stresses anisotropy caused by temperature gradient. Increase in height of the broken surface zone could be explained by the melt motion, pores formation and phase shifts. The conducted experiment at the above-described characteristics of both medium and laser has resulted in revealing that the role of the oxide layer in the formation of surface morphology is negligible. 
Experiment 2. MEDIUM=high vacuum $\left(p=2 \cdot 10^{-4} \mathrm{~Pa}\right) ; \quad$ TYPE=Nd: YAG laser $(\mathrm{ps})$; $\lambda=1064 \mathrm{~nm} ; \quad f=2 \mathrm{~Hz} ; \quad t=40 \mathrm{ps}$; $q=\{23.8 ; 40\} \mathrm{J} / \mathrm{cm}^{2}$. Brief results overview: both for a single pulse and for 5 laser pulses a crater with a smoothed center relief is formed on the Ti-surface. At pulse number equal to 30 , the nano-sized elements of morphology become noticeable and recrystallized titanium droplets are formed at the crater periphery due to the hydrodynamic effect. During the experiment, it has been found out that for the emergence of destruction effect of Ti-surface (i.e. for the crater formation) a minimum energy density of $0.9 \mathrm{~J} / \mathrm{cm}^{2}$ is required, and the crater depth strongly depends on the laser pulse number: for a single pulse the depth of the crater is equal to $50 \mu \mathrm{m}$, and for the pulse number of 5 is equal to $240 \mu \mathrm{m}$ (ablation rate in both cases is around $48 \mu \mathrm{m} /$ pulse). At more than 30 pulses and laser energy density of $q=40 \mathrm{~J} / \mathrm{cm}^{2}$, micro-cracks and nanograins are formed (which morphological character is caused by rapid heating and cooling processes), and the hydrodynamic effects at the edge of the crater are weaker. At a laser energy density of $q=23.8 \mathrm{~J} / \mathrm{cm}^{2}$, the absorbed energy exceeds titanium melting temperature threshold and melts appear in the crater center, which is pushed out on the crater periphery.

Experiment 3. MEDIUM=low vacuum $(p=13.3 \mathrm{~Pa}) ; \quad$ TYPE$=\mathrm{Ti}: \quad \mathrm{Al}_{2} \mathrm{O}_{3} \quad$ laser $\quad(\mathrm{fs})$; $\lambda=800 \mathrm{~nm} ; \quad f=1 \mathrm{kHz} ; \quad t=100 \mathrm{fs}$; $q=0.25 \div 1.5 \mathrm{~J} / \mathrm{cm}^{2}$. Brief results overview: the purpose of this experiment was to study the possibility of creating periodic structures on the polished Ti-surface (it is necessary to supplement the model (1)-(3) with the mathematical models described in the articles [25], [26]). During the experiment, it was found that at a constant laser energy density of $q=0.75 \mathrm{~J} / \mathrm{cm}^{2}$, the formed periodic structures morphology depends substantially on the number of laser pulses. In addition, it has been found out that, using the described environment and laser characteristics the minimum laser pulse number, which is required to form a periodic microstructure on the Ti-surface is equal to 10 (in the range $\left.q \in[0.25,1.5] \mathrm{J} / \mathrm{cm}^{2}\right)$. It has been found that in all cases the resulting structures are parallel to the electric polarization vector of the laser. At more than 50 laser pulses, micro-droplets are formed on the vertices of parallel periodic structures. Change in the number of pulses within the range of 10-25 leads to periodic nanostructures formation, which are combined with microstructures.

Experiment 4. MEDIUM=indoor atmosphere $(p=0.1 \mathrm{MPa}) ; \quad$ TYPE=ArF-eximer laser (ns); $\lambda=193 \mathrm{~nm} ; f=10 \mathrm{~Hz} ; t=18 \mathrm{~ns} ; q=1.5 \div 5 \mathrm{~J} / \mathrm{cm}^{2}$ (energy in pulse: $E=100 \mathrm{~mJ}$ ). Brief results overview: the purpose of this experiment was to study the possibility of polishing the Ti-surface at room indoor environment conditions. To form a texture on the Ti-surface, the laser energy density was increased to $q=8.5 \mathrm{~J} / \mathrm{cm}^{2}$, and the number of laser pulses varied from 250 to 1000 . It has been found out that effective Ti-surface polishing occurs only during $q \in[3,5] \mathrm{J} / \mathrm{cm}^{2}:$ the treatment completely eliminates traces of machining on the Ti-surface and substantially smoothens the surface, the roughness amplitude decreases from $256 \mu \mathrm{m}$ to $25 \mu \mathrm{m}$, and the root cause for this smoothing is either caused by surface layer melting and crystallization processes or by local heating and evaporation of the ledges under laser impacts. X-ray photoelectron spectroscopy revealed that the thickness of the oxide layer has been doubled.

\section{CONCLUSION}

This paper contains discusses about a relatively new direction related to the use of laser technology for surface treatment of biocompatible titanium implants in order to optimize its topography and physico-chemical properties. The brief overview of some of the results given in this paper demonstrate that laser treatment seems promising both in terms of providing a higher quality of the micromorphology of the surface of the material and in terms of increasing their corrosion resistance. It should be noted that the work aimed at improving the physical and chemical properties of the surface of titanium implants by laser treatment is still at the initial stage of its development within the framework of application in orthopedics. For a deeper understanding of the nature of the change in surface properties under the influence of laser treatment, further investigations are needed with the use of modern surface-sensitive analytical methods that provide quantitative information at the atomic-molecular level.

In addition, in this paper it is proposed a mathematical model in terms of spherical trigonometry for describing the transfer of laser radiation in the surface treatment of the titanium head of the human hip joint endoprosthesis.

\section{ACKNOWLEDGMENTS}

For the second co-author the present article was executed within the framework of The State Research Programme "Next generation Information and Communication Technologies" ("NextIT"), Project No. 4.

\section{REFERENCES}

[1] A. V. Puz, "Multifunctional Coatings for alloys of medical purpose", Doctoral Thesis, Institute of Chemistry, Far Eastern Department of the Russian Academy of Sciences, 2014, 164 p. 
Environment. Technology. Resources, Rezekne, Latvia Proceedings of the $11^{\text {th }}$ International Scientific and Practical Conference. Volume III, 350-356

[2] L. L.Hench, "Bioceramics", Journal of the American Ceramic Society, vol. 81, pp. 1705-1728, 1998.

[3] P. Cardaioli, M. Giordan, and G. Rigatelli, "Nickel allergy in interatrial shunt device-based closure patients", Journal of Congenital Heart Disease, vol. 2, No. 6, pp. 416-420, 2007.

[4] A. L. Gintsburg, A. S. Karyagina, and A. S. Semikhin, "Develepment of new generation of specimens for efficient repair of bone tissue", Journal of Therapy and Prophylaxis, No. 1, pp. 80-84, 2011.

[5] S. Takemoto S., Y. Kusudo, K. Tsuru, and S. Hayakawa, "Selective protein adsorption and blood compatibility of hydroxy-carbonate apatites", Journal of Biomedical Materials Research, vol. 69A, No. 3, pp. 544-551, 2004.

[6] R. S. Turmanidze, T. S. Aptsiauri, and G. Z. Popkhadze, "New Materials for Implants of the Human Hip Joint and Technology of Their Machining With the Achievement of High Precision and Quality of Spherical Surfaces", Journal of Mechanical Engineering, vol. 75, No. 3, pp. 64-71, 2015.

[7] O. A. Rozenberg, S. V.Sokhan, V. V.Vozny, A. G.Mamalis, J. Gavlik, and D.-J. Kim, "Trends and Development in the Manufacturing of Hip Joints: An Overview", International Journal of Advanced Manufacturing Technology, vol. 27, pp. 537-542, 2006.

[8] M. A. Vasilev, M. M. Nischenko, and P. A. Gurin, "Laser modification of surface of titanium implants", Progresses of Physical Metallurgy, vol. 11, pp. 209-247, 2010

[9] M. M. Nischenko, N. A. Shevchenko, D. V. Schur, V. A. Bogolepov, A. G. Dubovoi, and I. M. Sidorchenko, "Laserstimulated emission of electrons from carbon nanostructures formed at laser evaporation of oriented carbon nanotubes", Journal of Inorganic Materials: Applied Research, vol. 1, No. 4, pp. 276-278, 2010.

[10] P.-I. Branemark, B. O. Hansson, R. Adell,U. Breine, J. Lindstrom, O. Hallen, and A. Ohman, "Osseointegrated implants in the treatment of the edentulous jaw. Experience from a 10-year period", Scandinavian Journal of Plastic and Reconstructive Surgery and Hand Surgery, vol. 16, pp. 1-132, 1977

[11] R. Adell, U. Lekholm, B. Rockler, and P.-I. Branemark, "A 15-year study of osseointegrated implants in the treatment of the edentulous jaw", International Journal of Oral Surgey, vol 10, No. 6, pp. 387-416, 1981.

[12] "General statistics about papers of P.-I. Brånemark". [Online].

Available:

http://scicurve.com/author/Br\%C3\%A5nemark\%20P\%20I [Accessed: March 08, 2017].

[13] G. Wang and H. Zraigat, "Functional Coatings or Films for Hard-Tissue Applications", Journal of Materials, vol. 3, No. 7, pp. 3994-4050, 2010.
[14] "Titanium alloy". [Online]. Available: https://en.wikipedia.org/wiki/Titanium_alloy [Accessed: March 08, 2017].

[15] T. Traini, M. Berardini, F. Congedi, B. Sinjari, P. Trisi, and S. Caputi, "Impact of Second Stage Surgery on Bone Remodeling Around New Hybrid Titanium Implants: A Prospective Clinical Study in Humans", Journal of Implant Dentistry, vol. 26, No. 1, pp. 121-128, 2017.

[16] P. Trisi, M. Berardini, M. Colagiovanni, D. Berardi, and G. L. Perfetti, "Laser-Treated Titanium Implants: An In Vivo Histomorphometric and Biomechanical Analysis", Journal of Implant Dentistry, vol. 25, No. 5, pp. 575-580, 2016.

[17] M. A. Vasilev, S. P. Chenakin, M. M. Nishchenko, L. F. Yatsenko, "Effect of pulsed laser radiation to the composition of the surface layers of the titanium alloy VT6", Journal of Metal Physics and Advanced Technologies, vol 37, No. 7, pp. 861-870, 2015

[18] N. V. Novikov, O. A. Rozenberg, J. Gavlik, S. V. Sohan, V. V. Vozniy, R. S. Turmanidze, D. S. Buchrikidze, and M. D. Beridze, Implants of Human Joints: Materials and Technologies. Kiev, Ukraine: Bakul Institute for Superhard Materials, The National Academy of Sciences of Ukraine, 2011, 528 p.

[19] C. I. Palmer and C.W.Leigh, Plane and Spherical Trigonometry. New York \& London: McGraw-Hill Book Company, 1934, xiv+229 p.

[20] M. K. Ventsel, Spherical Trigonometry. Moscow, USSR: GeodezIzdat, 1934, $154 \mathrm{p}$

21] A. N. Tikhonov and V. Ya. Arsenin, Solution of Ill-posed Problems. Washington, USA: Winston \& Sons, 1977, xiii+258 p.

[22] S. A. Sarra, "The Method of Characteristics with applications to Conservation Laws", Journal of Online Mathematics and its Applications, vol. 3, pp. 1-16, 2003.

[23] M. Delgado, "The Lagrange-Charpit Method", SIAM Review, vol. 39, No. 2, pp. 298-304, 1997

[24] I. M. Gelfand, "Some problems of Theory of Quasi-Linear Equations, Advances of Mathematical Sciences (Russian Mathematical Surveys), vol. 14, No. 2, pp. 97-158, 1959.

[25] Sh. E. Guseynov and U. Zaimis, "On a physico-mathematical model for controlled formation of periodic nanostructures at solid surfaces irradiated by femtosecond laser pulses", Journal of Nonequilibrium Phase Transformations, No. 3/2016, pp. 17-20, 2016.

[26] Sh. E. Guseynov and U. Zaimis, "On a physico-mathematical model for controlled formation of periodic nanostructures at solid surfaces irradiated by femtosecond laser pulses", Journal of Machines, Technologies, Materials, No. 7/2016, pp. 23-26, 2016. 